\title{
GANGLIONEUROBLASTOMA OF THE RETROPERITONEUM: A RARE CASE REPORT
}

\author{
Ansar Pullampura Pookunju¹, Ramaswamy Rajendran², Prithviraj Premkumar³, Ayyapan Srinivasan4
}

${ }^{1}$ Senior Registrar, Department of Surgical Oncology, Apollo Speciality Hospitals, Chennai.

${ }^{2}$ Senior Trainee, Department of Surgical Oncology, Madras Medical College, Rajiv Gandhi Government General Hospital, Chennai. 3 Senior Trainee, Department of Surgical Oncology, Madras Medical College, Rajiv Gandhi Government General Hospital, Chennai. ${ }^{4}$ Head and Senior Consultant, Department of Surgical Oncology, Apollo Speciality Hospitals, Chennai.

\section{ABSTRACT}

Ganglioneuroblastoma is a neuroblastic tumour arising from primitive sympathetic ganglion cells. Neuroblastic tumours have a broad-spectrum of clinical behaviour from spontaneous regression to benign ganglioneuromas to metastatic dissemination. We present a case report of 2-year of girl who had a provisional diagnosis of an ovarian mass. Exploratory Laparotomy in a peripheral center revealed a retroperitoneal mass crossing the midline extending into the pelvis. She was unresectable and histopathology showed Ganglioneuroblastoma Stage III. She was managed with chemotherapy and repeat surgery for residual disease. Surgery remains the choice of treatment for neuroblastic tumours.

\section{KEYWORDS}

Neuroblastic tumour, Ganglioneuroblastoma, Retroperitoneal Neuroblastoma.

HOW TO CITE THIS ARTICLE: Pookunju AP, Rajendran R, Premkumar P, et al. Ganglioneuroblastoma of the retroperitoneum: a rare case report. J. Evolution Med. Dent. Sci. 2016;5(45):2843-2847, DOI: 10.14260/jemds/2016/664

\section{INTRODUCTION}

A spectrum of neuroblastic tumours (Neuroblastomas, ganglioneuroblastomas, ganglioneuromas) arising from the primitive sympathetic ganglion cells. They synthesize and secrete catecholamines. These tumours have a broadspectrum of behaviour from spontaneous regression to maturation to a benign ganglioneuroma or aggressive disease with metastatic dissemination leading to death. We present this case for its rarity, unusual location of the tumour and its extent and management of the tumour.

\section{CASE REPORT}

A 2-year-old girl presented with intermittent episodes of abdominal pain and distension for 6 months duration, parents noticed diffuse swelling in the lower abdomen and increased frequency of micturition, she was evaluated with USG abdomen followed by a contrast enhanced CT scan which showed a heterogeneous mass in the retroperitoneum which crosses the midline and extending into the pelvis (Figure 1). There was mild enhancement with the contrast. Serum markers AFP, HCG, LDH were normal. A provisional diagnosis of ovarian origin was made and exploratory laparotomy was done in the peripheral centre. Findings reported were a Firmto-Hard mass originating in the retroperitoneal region (ParaAortic) crossing the midline and extending into the pelvis encircling the iliac vessels on the left side. Incomplete resection was done due to vascular structure encasement.

Histopathology-Ganglioneuroblastoma of Intermixed type and International neuroblastoma Staging System-Stage 3 Chest X-ray, Bone Marrow Biopsy, Peripheral Smear, Serum Catecholamine and its metabolites were normal, Vanillylmandelic Acid (VMA) in the urine was normal.

Financial or Other, Competing Interest: None.

Submission 21-04-2016, Peer Review 14-05-2016,

Acceptance 21-05-2016, Published 06-06-2016.

Corresponding Author:

Dr. Ansar Pullampura Pookunju,

\#C1 Triveni,

62 Alwarpet Street

Chennai-18.

E-mail:dr.ansarpp@gmail.com

DOI: $10.14260 /$ jemds/2016/664
Post-operative adjuvant chemotherapy was given according to the Children's Cancer Group (CCG) Protocol with Cyclophosphamide, Cisplatin and Adriamycin for 6 cycles. On reassessment with CECT, residual tumour was identified and she was referred to our centre.

CT Images showed a mildly enhancing residual tumour in the retroperitoneum and pelvis crossing the midline and pushing the left iliac vessels anteriorly (Figure 2). After Tumour Board discussion, exploratory laparotomy through the previous transverse laparotomy scar was performed (Figure 4). Adhesiolysis, rectosigmoid colon mobilization was done first to expose the retroperitoneum following which the tumour was dissected away from iliac vessels and removed Monobloc (Figure 5).

Adjuvant chemotherapy was given to the patient. At 6 months followup, she was recurrence free. No radiation was considered because of complete excision.
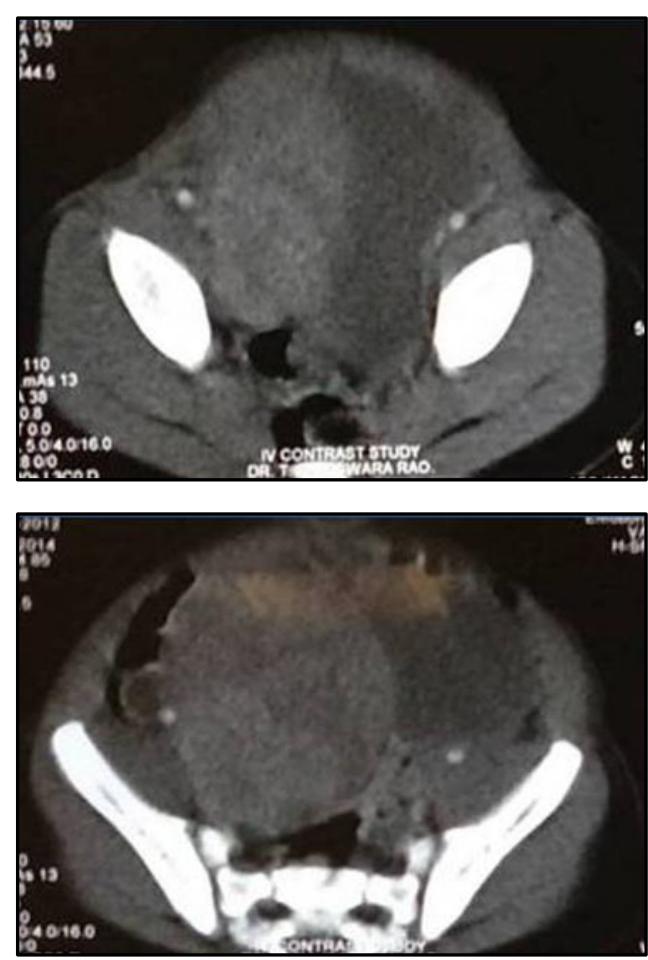


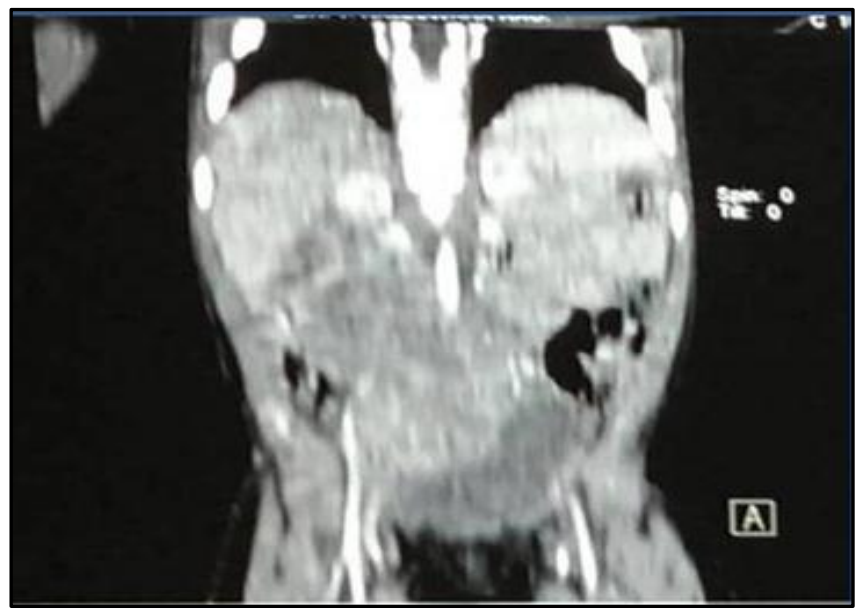

Fig. 1: Heterogeneous Mass in the Retroperitoneum Extending into the Pelvis Mild Enhancement with the Contrast is Made Out

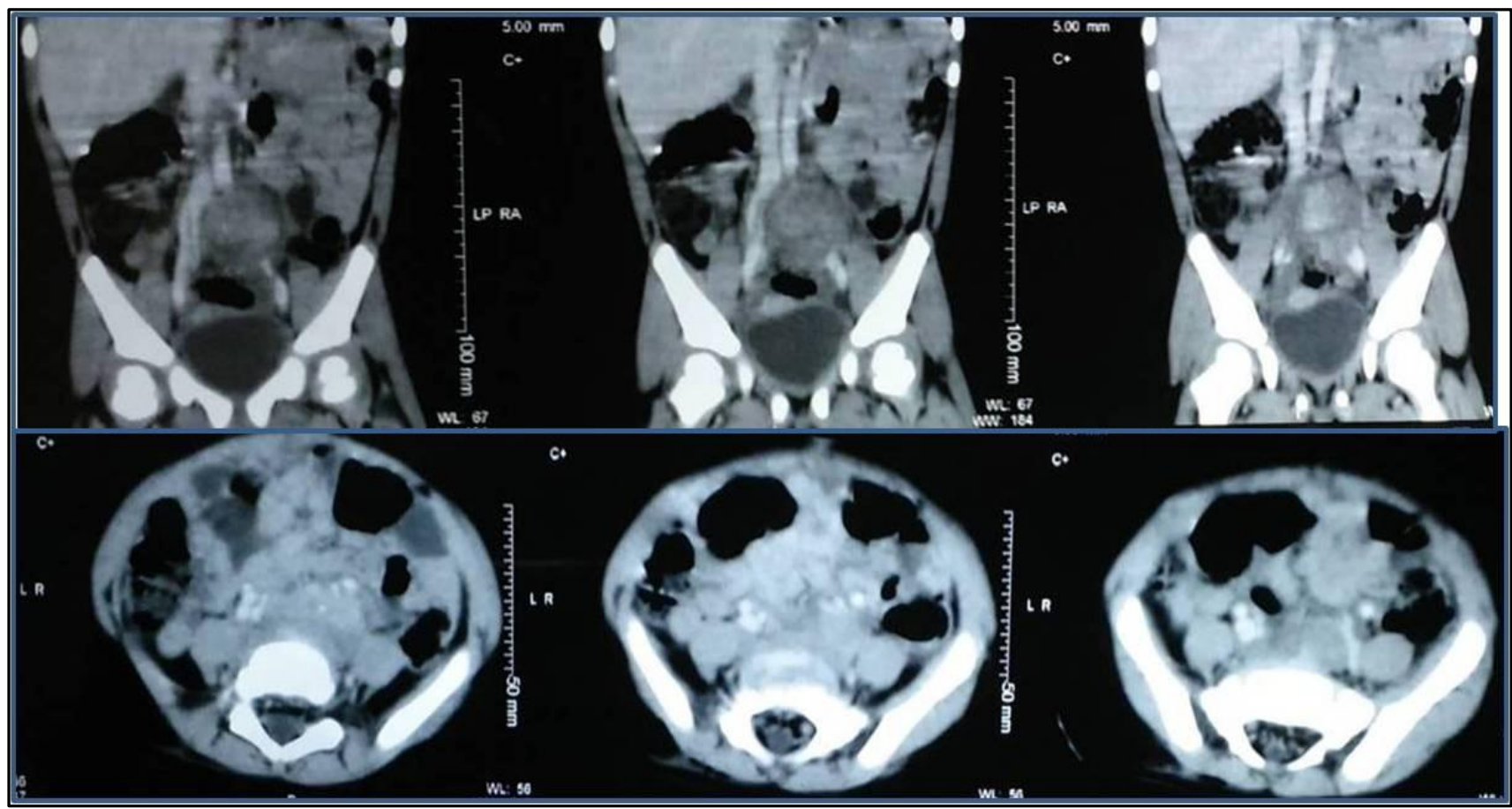

Fig. 2: After Debulking Surgery (R2 Resection) and Adjuvant Chemotherapy there is Enhancing Residual Tumour in the Retroperitoneum (Para-aortic) Extending into the Pelvis Displacing the Left Iliac Vessels Anteriorly

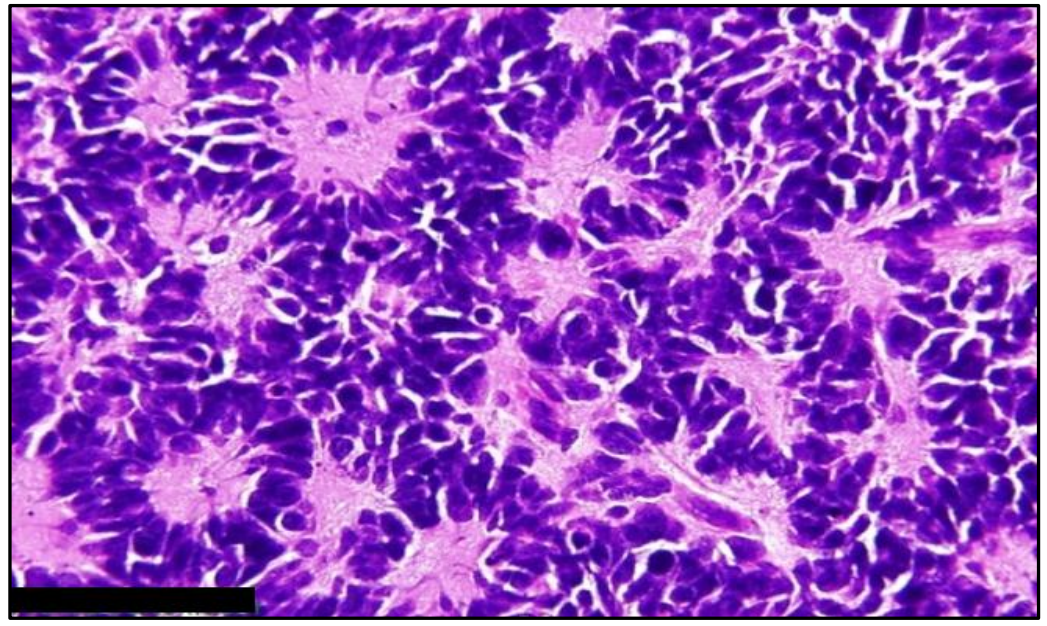

Fig. 3: Homer Wright Rosettes Are Circular Groupings of Dark Tumour Cells Surrounding Pale Neurofibrils (Small Blue Cell Tumours from Neural Crest Ectoderm) 


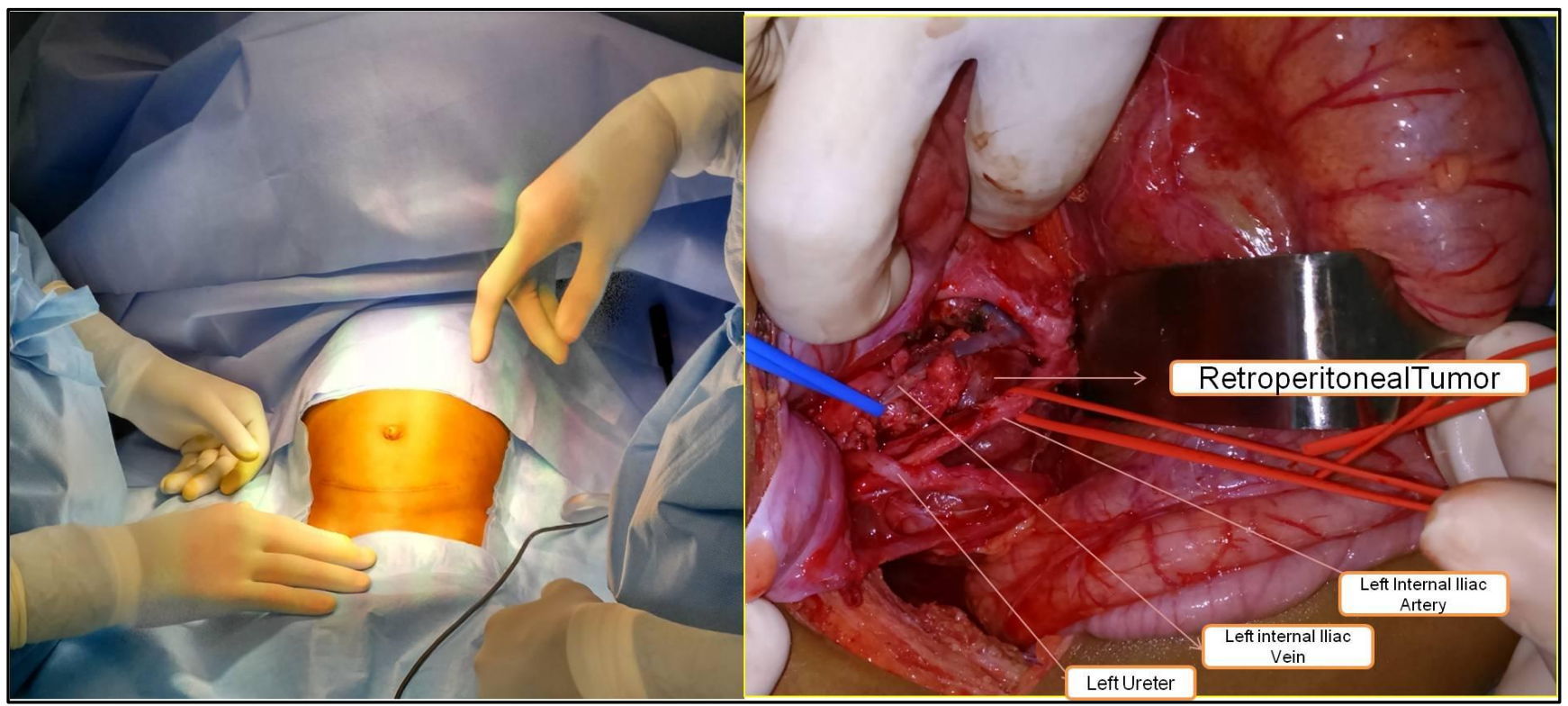

Fig. 4: Exploratory Laparotomy Done Through Previous Transverse Laparotomy Scar and Intraoperative Image shows the Tumour Located Behind and Displacing the Left Iliac Vessels and the Left Ureter

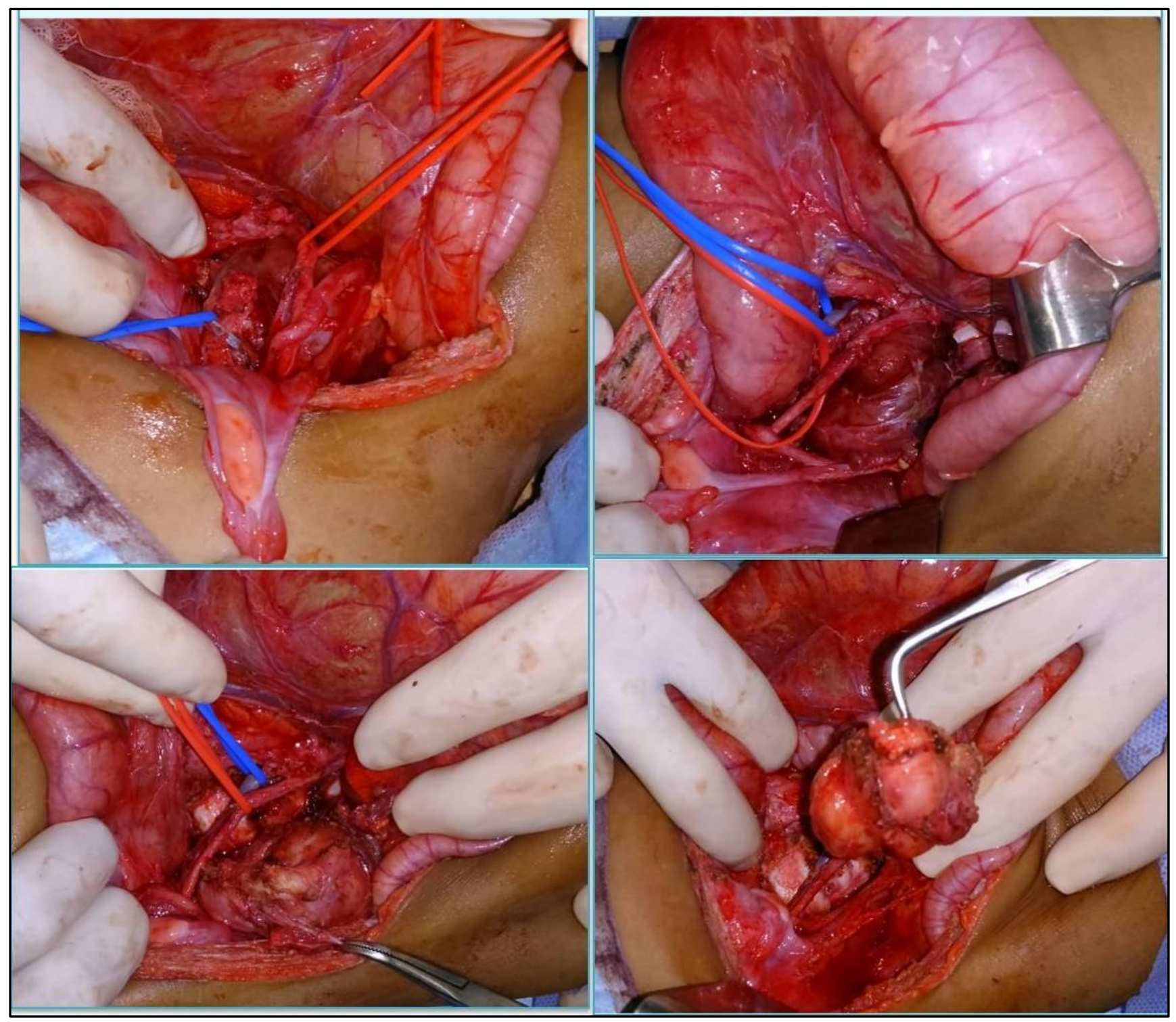

Fig. 5: Rectosigmoid Colon Mobilization to Expose the Retroperitoneum. The Left Ureter and Left Iliac Vessels We Carefully Dissected Away from the Tumour and the Tumour was Removed Monobloc 


\section{DISCUSSION}

Neuroblastoma constitutes $5 \%$ of solid tumour. Adrenal Gland $(40 \%)$ is the most common site for primary, Abdominal (25\%), Thoracic (15\%), Cervical (5\%), Pelvic sympathetic ganglia (5\%). It can occur in both Central nervous system and Autonomic nervous system. Neuroblastic tumours form 8 to $10 \%$ of all childhood cancers; 10 cases per one million live births and is the most common malignant tumour of the infancy. The median age of diagnosis is at 19 months. There are no geographic or racial variations. It can occur as Sporadic, hereditary and familial inheritance. It is an Autosomal dominant pattern of inheritance. Hereditary neuroblastoma predisposition gene is in chromosome 16p1213. Amplification of the N-MYC Oncogene seen in roughly $20 \%$ and Deletion of the short arm of chromosome 1 . In familial cases, median age of diagnosis decreases to 9 months.

The histopathological examination of neuroblastoma reveals a tumour with undifferentiated, primitive-appearing round blue cells with hyperchromatic nuclei and scant cytoplasm in a lobular pattern. Sheets of Homer-Wright pseudorosettes (Figure 3) consisting of neuroblasts surrounding eosinophilic neuropil, are a pathognomonic feature confirming the diagnosis of neuroblastoma.(1-3)

These tumours were originally classified according to a classification system by Shimada et al, which was subsequently clarified and adopted for use globally by The International Neuroblastoma Pathology Committee-INPC System 11 proposed in 1999 and revised in 2003.

Based on the balance between the neural type cells and Schwannian type cells and Schwannian stroma, these tumours are classified into four histologic subtypes: (1) Neuroblastoma (Schwannian stroma-poor), undifferentiated, poorly differentiated and differentiating; Ganglioneuroblastoma, intermixed (Schwannian stromarich); (3) Ganglioneuroma (Schwannian stroma dominant), maturing and mature; and (4) Ganglioneuroblastoma, nodular (Composite Schwannian stroma rich/stroma dominant and stroma poor).

Neuroblastoma tumours are classified into Favourable Histology (FH) and Unfavourable Histology (UH) lesions based on the degree of stromal development, morphology, (Mitosis and karyorrhexis) MKI and age.

The gene that was consistently amplified in these segments was identified by Schwab and Colleagues.(4) as a myc-related gene, which was eventually called MYCN (v-myc avian myelocytomatosis viral oncogene neuroblastomaderived homolog). Amplification of MYCN is predominantly associated with advanced stage disease, rapid tumour progression, and poor prognosis. (5)

It is a catecholamine secreting tumour and the clinical features are of wide range from Abdominal pain, Palpable fixed hard abdominal mass, Bone or joint pain, Periorbital ecchymosis, Cough, Dyspnoea, Neurologic deficits, Urinary retention, Constipation, Paraneoplastic syndromes, Paroxysmal hypertension, Palpitations, Flushing, Headache, Severe watery diarrhoea, Hypokalaemia, Acute myoclonic encephalopathy.

Laboratory investigations of value are serum Catecholamine levels and Urine HVA and VMA excretion $>3$ SD above mean per milligram Creatinine for age; $80 \%$ of the neuroblastoma has increased VMA, HVA in urine. Bone marrow biopsy or aspiration is done to identify bone marrow involvement; Immunohistochemistry staining with synaptophysin or chromogranin are done to confirm the presence of neuroblasts if the diagnosis is based on the bone marrow aspirate.

Ultrasound is often the initial radiologic study to identify the tumour. However, Magnetic Resonance Imaging (MRI) or Computerized Tomography (CT) of the chest, abdomen and pelvis is required to clearly define the location and extent of the primary tumour and its relations to major vessels. Radiographically, a neuroblastoma typically appears as a heterogeneous mass with calcifications. Adrenal and retroperitoneal tumours characteristically involve and displace the major vessels.

Meta-iodobenzylguanidine (MIBG) scan is done for all patients at the time of diagnosis and has replaced technetium $99 \mathrm{~m}$ bone scan. MIBG scan has $90 \%$ sensitivity and $100 \%$ specificity. Receptor proteins for the MIBG are expressed by 90\% of neuroblastoma. 18F-FDG, 18F-DOPA Positron Emission Tomography can be considered in $10 \%$ patients who are negative for MIBG.

\section{International Neuroblastoma Staging System.(6)}

Stage I-localized tumour with complete gross excision without microscopic residual disease; representative ipsilateral lymph nodes negative for tumour microscopically.

Stage IIA-localized tumour with incomplete gross excision; representative ipsilateral non-adherent lymph nodes negative for tumour microscopically.

Stage IIB-localized tumour with or without complete gross excision with ipsilateral non-adherent lymph nodes positive for tumour. Enlarged contralateral lymph nodes must be negative microscopically.

Stage III-unresectable unilateral tumour infiltrating across midline, with or without regional lymph node involvement; or localized unilateral tumour with contralateral regional lymph node involvement; or midline tumour with bilateral extension by infiltration (Unresectable) or by lymph node involvement.

Stage IV-any primary tumour with dissemination to distant lymph nodes, bone, bone marrow, liver, skin or other organs.

Stage IVS-localized primary tumour as defined for stage I, IIA or IIB with dissemination limited to skin, liver or bone marrow.

Staging along with age at diagnosis and certain tests on tumour specimens, such as histology and $\mathrm{N}$ myc amplification, play critical roles in the recommendations of specific treatment modalities and evaluates the risk of recurrence.(6)

Children's Oncology Group's (COG) risk categories incorporate the patient's age at presentation, stage of the tumour, histological appearance, quantitative DNA content, and presence or absence of MYCN amplification within the tumour cells. It classifies the patients into low, intermediate and high risk categories.

Studies have demonstrated that in low risk group total surgical excision is curative in $>95 \%$ of patients. Resectability of the tumour depends on the tumour location with relationship to major blood vessels and nerves, tumour mobility, age and metastasis.

Role of chemotherapy is reserved for unresectable tumours where the tumour is downsized and made amenable 
for surgery and in oncologic emergencies like spinal cord compression. Cyclophosphamide, doxorubicin, carboplatin, and etoposide are the commonly used drug combination. [7-14]

Autologous Stem Cell Transplant (ASCT) for neuroblastoma has been performed using various combinations of chemotherapy with or without Total Body Irradiation (TBI). Whenever there is failure to control the primary tumour, the primary radiation has been used as a part of consolidation therapy.(15) In circumstances of soft tissue and bone metastasis of advanced disease, radiation therapy is used to control pain.

\section{CONCLUSION}

Rare occurrence of Ganglioneuroblastoma in paediatric population should always be kept as a differential diagnosis for evaluation whenever a young child presents with a symptomatic mass as in our case. More than $50 \%$ of the tumours are low risk group and has overall survival greater than $95 \%$ with surgical treatment. Our patient was a stage III with intermediate risk disease. She underwent chemotherapy for downsizing the lesion followed by surgical removal. Patient is on a close followup and now recurrence free for 6 months.

\section{REFERENCES}

1. Weinstein JL, Katzenstein HM, Cohn SL. Advances in the diagnosis and treatment of neuroblastoma. The Oncologist 2003;8(3):278-92.

2. Triche TJ, Askin FB. Neuroblastoma and the differential diagnosis of small-, round-, blue-cell tumours. Hum Pathol 1983;14(7):569-95.

3. Rubie H, Hartmann $\mathrm{O}$, Michon J, et al. N-myc gene amplification is a major prognostic factor in localized neuroblastoma: results of the French NBL 90 study neuroblastoma study group of the société française d'oncologie pédiatrique. J Clin Oncol 1997;15(3):117182.

4. Schwab M, Alitalo K, Klempnauer KH, et al. Amplified DNA with limited homology to myc cellular oncogene is shared by human neuroblastoma cell lines and a neuroblastoma tumour. Nature 1983;305:245-8.

5. Brodeur GM, Seeger RC, Schwab M, et al. Amplification of $\mathrm{N}$-myc in untreated human neuroblastomas correlates with advanced disease stage. Science 1984;224(4653):1121-4.
6. Brodeur GM, Pritchard J, Berthold F, et al. Revisions of the international criteria for neuroblastoma diagnosis, staging, and response to treatment. J Clin Oncol 1993;11(8):1466-77.

7. Katzenstein HM, Bowman LC, Brodeur GM, et al. Prognostic significance of age, MYCN oncogene amplification, tumour cell ploidy, and histology in 110 infants with stage $D(S)$ neuroblastoma: the paediatric oncology group experience-a paediatric oncology group study. J Clin Oncol 1998;16(6):2007-17.

8. Nickerson HJ, Matthay KK, Seeger RC, et al. Favourable biology and outcome of stage IV-S neuroblastoma with supportive care or minimal therapy: a children's cancer group study. J Clin Oncol 2000;18(3):477-86.

9. Simon T, Spitz R, Faldum A, et al. New definition of lowrisk neuroblastoma using stage, age, and $1 \mathrm{p}$ and MYCN status. J Paediatr Hematol Oncol 2004;26(12):791-6.

10. Brodeur GM, Maris JM. Neuroblastoma. In: principles and practice of paediatric oncology. Pizzo PA, Poplack DG, eds. Philadelphia: Lippincott Williams, Wilkins, 2002:895-938.

11. Laprie A, Michon J, Hartmann O, et al. High-dose chemotherapy followed by locoregional irradiation improves the outcome of patients with international neuroblastoma staging system stage II and III neuroblastoma with MYCN amplification. Cancer 2004;101(5):1081-9.

12. Haas-Kogan DA, Swift PS, Selch M, et al. Impact of radiotherapy for high-risk neuroblastoma: a children's cancer group study. Int J Radiat Oncol Biol Phys 2003;56(1):28-39.

13. Marcus KJ, Shamberger R, Litman H, et al. Primary tumour control in patients with stage $3 / 4$ unfavourable neuroblastoma treated with tandem doubleautologous stem cell transplants. J Paediatr Hematol Oncol 2003;25(12):934-40.

14. Rowe PH, Oram JJ, Scott GW. Neuroblastoma in adults. Postgrad Med J 1979;55(646):579-80.

15. Matthay KK, Villablanca JG, Seeger RC, et al. Treatment of high-risk neuroblastoma with intensive chemotherapy, radiotherapy, autologous bone marrow transplantation, and 13-cis-retinoic acid. Children's Cancer Group N Engl J Med 1999;341:1165-73. 